\title{
Challenging Case: Two Successive Unusual Causes of Revision Hip Prosthesis; Management and Outcome
}

\author{
Naoufal Elghoul ${ }^{*}$, Tariq Hamamouch ${ }^{2}$, Laribi Adam², Georges Saad², Mohammed \\ Zaaf and Abdeloihab Jaafar ${ }^{4}$
}

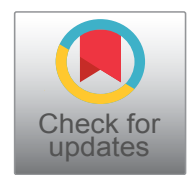

${ }^{1}$ Resident in Orthopedic Surgery and Traumatology, Department of Orthopedic Surgery and Traumatology, Faculty of Medicine and Pharmacy, Military Hospital Mohammed V, Mohammed V University of Rabat, Morocco

${ }^{2}$ Hospital Practitioner in Orthopedic Surgery and Traumatology, Centre Hospitalier Sud Essonne Dourdan, Etampes, France ${ }^{3}$ Chief of Department of Surgery, Hospital Practitioner in Orthopedic Surgery and Traumatology, Centre Hospitalier Sud Essonne Dourdan, Etampes, France

${ }^{4}$ Chief and Professor in Orthopedic Surgery and Traumatology, Faculty of Medicine and Pharmacy, Military Hospital Mohammed V, Mohammed V University of Rabat, Morocco

*Corresponding author: Dr. Naoufal Elghoul, Resident in Orthopedic Surgery and Traumatology, Department of Orthopedic Surgery and Traumatology, Faculty of Medicine and Pharmacy, Military Hospital Mohammed V, Mohammed V University of Rabat, BP 10100, Morocco

\begin{abstract}
Introduction: Femoral stem fracture is an extremely rare cause, especially in cementless nonmodular stem femoral. Furthermore, revision surgery for isolated polyethylene wear represents a challenge and dilemma for the orthopedic surgeon. This study aimed to present the first case that described two successive challenging causes of total hip arthroplasty revision, and to detail how they were how they were best managed.
\end{abstract}

Case presentation: A 65-year-old male patient with a body mass index of 31 who underwent a primary left cementless total hip arthroplasty for the end-stage arthritic hip. 4 years later, he presented a femoral stem fracture diagnosed following the clinical and radiological findings prompting the patient to undergo surgery. Only the femoral component was revised with good results for 5 years. After that, he gradually presented a pain of the left hip with clinical lameness. The radiograph of the hip showed that the femoral head was embedded in the acetabulum component, and so revision surgery was indicated. The intraoperative aspect found a total wearing of the acetabulum's polyethylene then a dual-mobility acetabular component was used while the femoral stem prosthesis was conserved. At a follow-up of 2 years, the patient was pain-free and could ambulate with no assistive devices and no leg length inequality.

Conclusion: However, implant fracture is extremely rare; it can be highly traumatizing for patients. The isolated polyethylene wear remains a serious problem for engineers who manufacture prostheses, for surgeons who implant them and for patients who get the result.

\section{Keywords}

Implant fracture, Polyethylene wear, Revision surgery, Hip prosthesis

\section{Introduction}

Total hip arthroplasty (THA) constitutes currently the cornerstone of surgical management of end-stage arthritic hip conditions [1,2]. The number of patients operated for hip prosthesis has increased substantially over the past several years. At least, this number will be more than 572,000 by the year 2030 [3]. On the other hand, it is known that "the more prosthesis we implant, the more revision surgery, we will perform" that's why, also, the number of revision hip procedures is expected to increase to 96,700 by 2030 [4]. This revision surgery is due to many causes such as periprosthetic fracture, infection, osteolysis. But, implant fracture as one of the modes of failure of total hip arthroplasty is an extremely rare cause, especially in cementless nonmodular stem femoral $[5,6]$. More-

Citation: Elghoul N, Hamamouch T, Adam L, Saad G, Zaaf M, et al. (2020) Challenging Case: Two Successive Unusual Causes of Revision Hip Prosthesis; Management and Outcome. Int Arch Orthop Surg 3:017. doi.org/10.23937/2643-4016/1710017

Accepted: February 22, 2020; Published: February 24, 2020

Copyright: (c) 2020 Elghoul N, et al. This is an open-access article distributed under the terms of the Creative Commons Attribution License, which permits unrestricted use, distribution, and reproduction in any medium, provided the original author and source are credited. 
over, revision surgery for isolated polyethylene wear represents a challenge and dilemma for the orthopedic surgeon [7].

To the best of our knowledge, this is the first such case to be reported in the literature that described two successive challenging causes of total hip arthroplasty revision.

\section{Case Report}

A 65-year-old male patient weighed $94 \mathrm{~kg}$ with a body mass index (BMI) of 31, had been diagnosed with osteoarthritis of both hip since 12 years ago; the left side was the more painful (Figure $1 \mathrm{~A}$ ) reason for which he underwent a primary left cementless total hip arthroplasty (Valmer Cup size 50; SILENE femoral stem size 5; ceramic femoral head with long size $28 \mathrm{~mm}$ ) (Figure 1B)). A Hardinge external approach was performed with neither reported intraoperative nor postoperative complications. The patient did well (no pain of left hip and a tolerated pain of right hip) until 2011 (4 years later) at which time he reported an insidious thigh pain in the left hip, got worse gradually with weight-bearing, unrelieved by medication; his only relief came from rest and restriction of weight-bearing. Two weeks later, when the patient was getting up from a sitting position brutally, he heard a broken sound in his left hip that caused a painful hip movement. Then he was transferred to the emergency department. On Admission, he was in good general health with no distress signs. Physical examination found both palpation and mobilization of the left hip were painful; the homolateral leg was shortened and externally rotated. The neurovascular examination was normal, and the skin of the operative site was good. Emergency radiographs of the pelvis showed a rupture at the neck-stem junction of the femoral component with no associated fracture or bone losing (Figure 2) prompting the patient to undergo surgery. Thus, three days later, after anesthetic preparation, he was admitted to the operating room for revision surgery via the same surgical approach. Intraoperative aspects showed a fracture of the femoral stem; since the extraction of the remainder femoral stem did not seem feasible, we performed a femoral osteotomy; it was removed after making it free; the acetabulum was well fixed with no evidence of scratches or loosening and so the cup was not revised. A multiple stainless steel cerclage wires
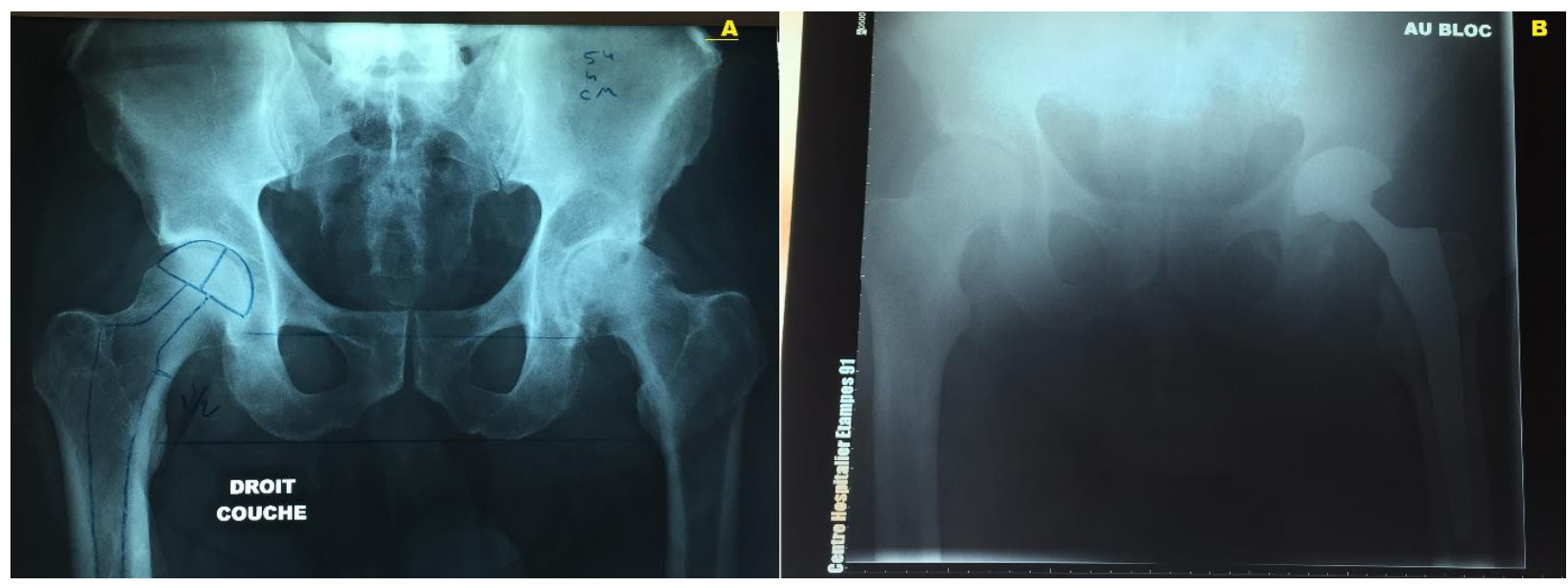

Figure 1: A) Anterior-posterior pelvic x-ray showed advanced left hip osteoarthritis (Femoral offset $=41 \mathrm{~mm}$ ); B) On postoperative $x$-ray control, Femoral offset $=38 \mathrm{~mm}$, cap inclination $=33^{\circ}$.
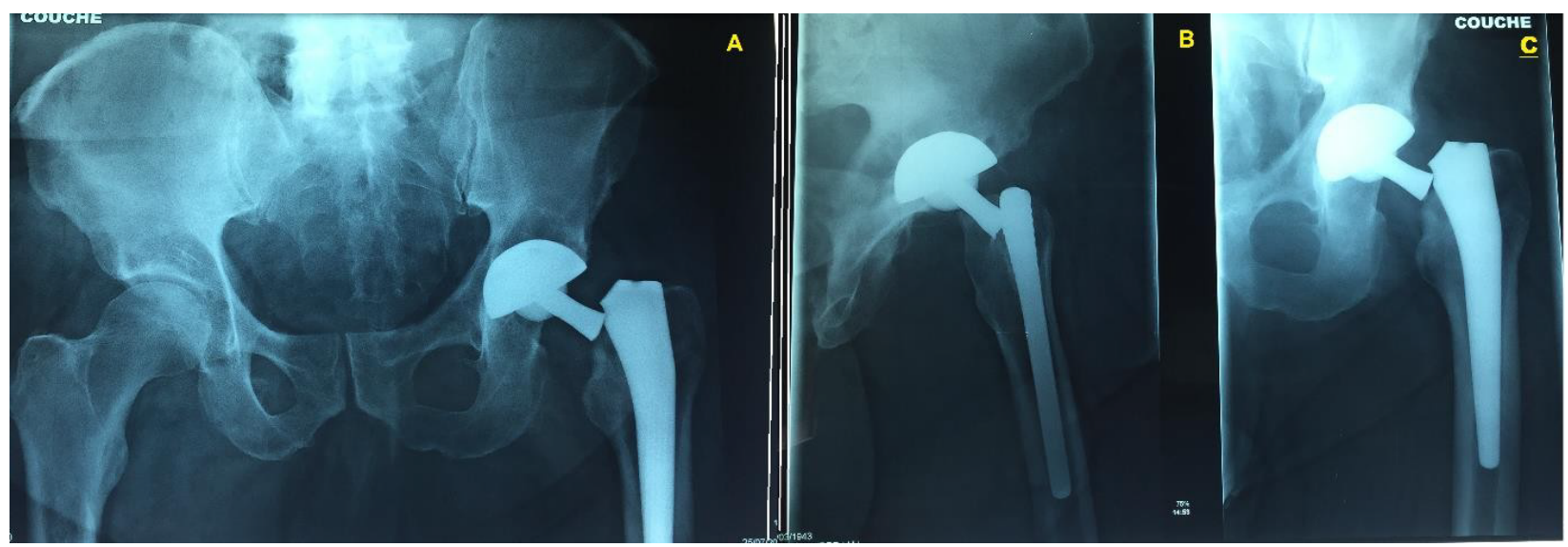

Figure 2: Emergency radiographs (anterior-posterior views of the pelvis $(A)$, of the hip $(C)$ and lateral view of the hip (B) showed a rupture at the neck-stem junction of the cementless nonmodular stem femoral with neither associated fracture nor bone losing. 
were inserted to secure extended trochanteric osteotomy (Figure 3): Implanted components included a fem- oral revision stem cemented size 4 and $28 \mathrm{~mm}$ ceramic femoral head with a medium neck. Hip exercises such as

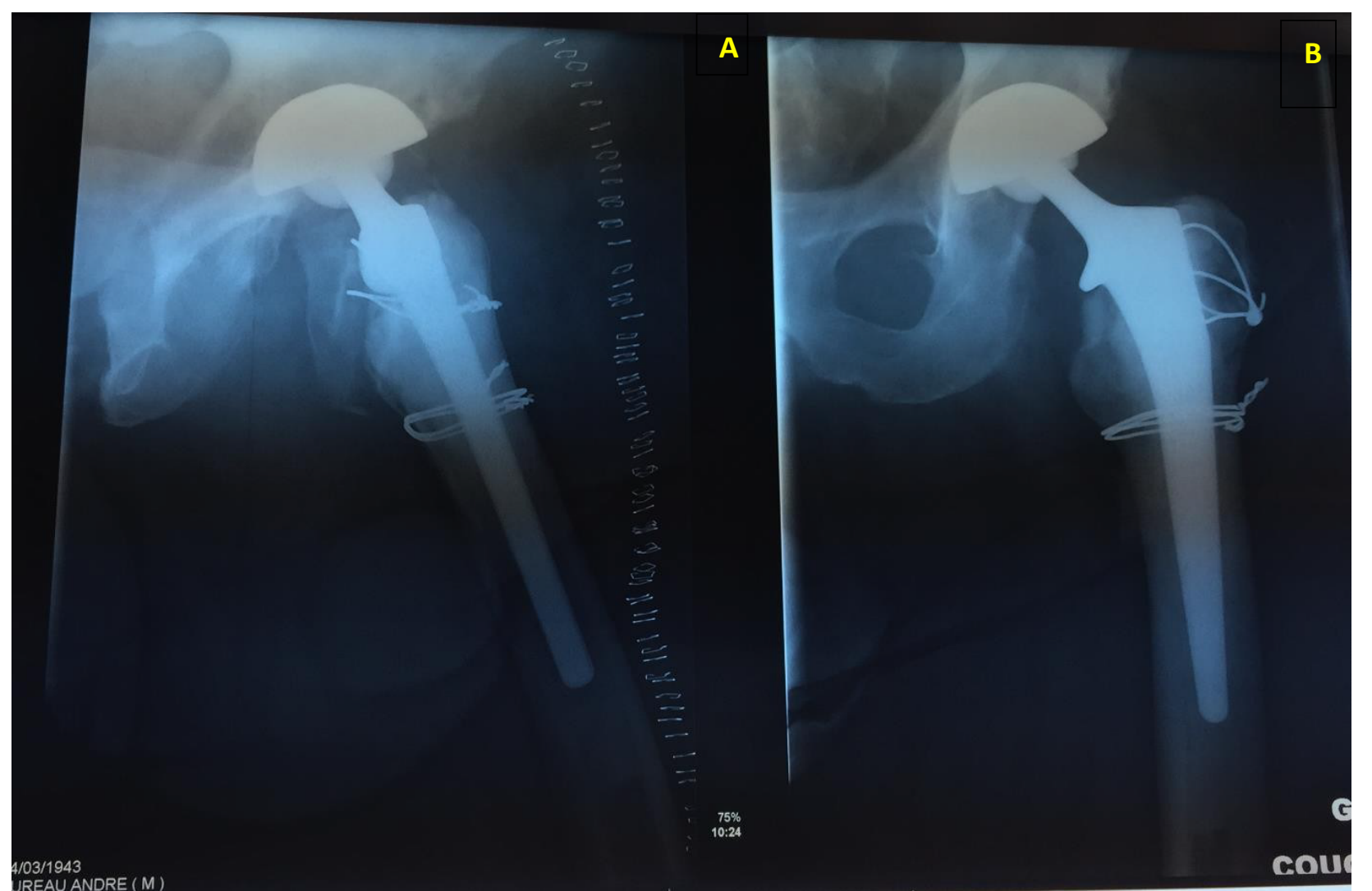

Figure 3: The post-operative X-rays of the left hip ( lateral $(A)$ and anterior-posterior $(B)$ views ) showed a good fixation of the components.
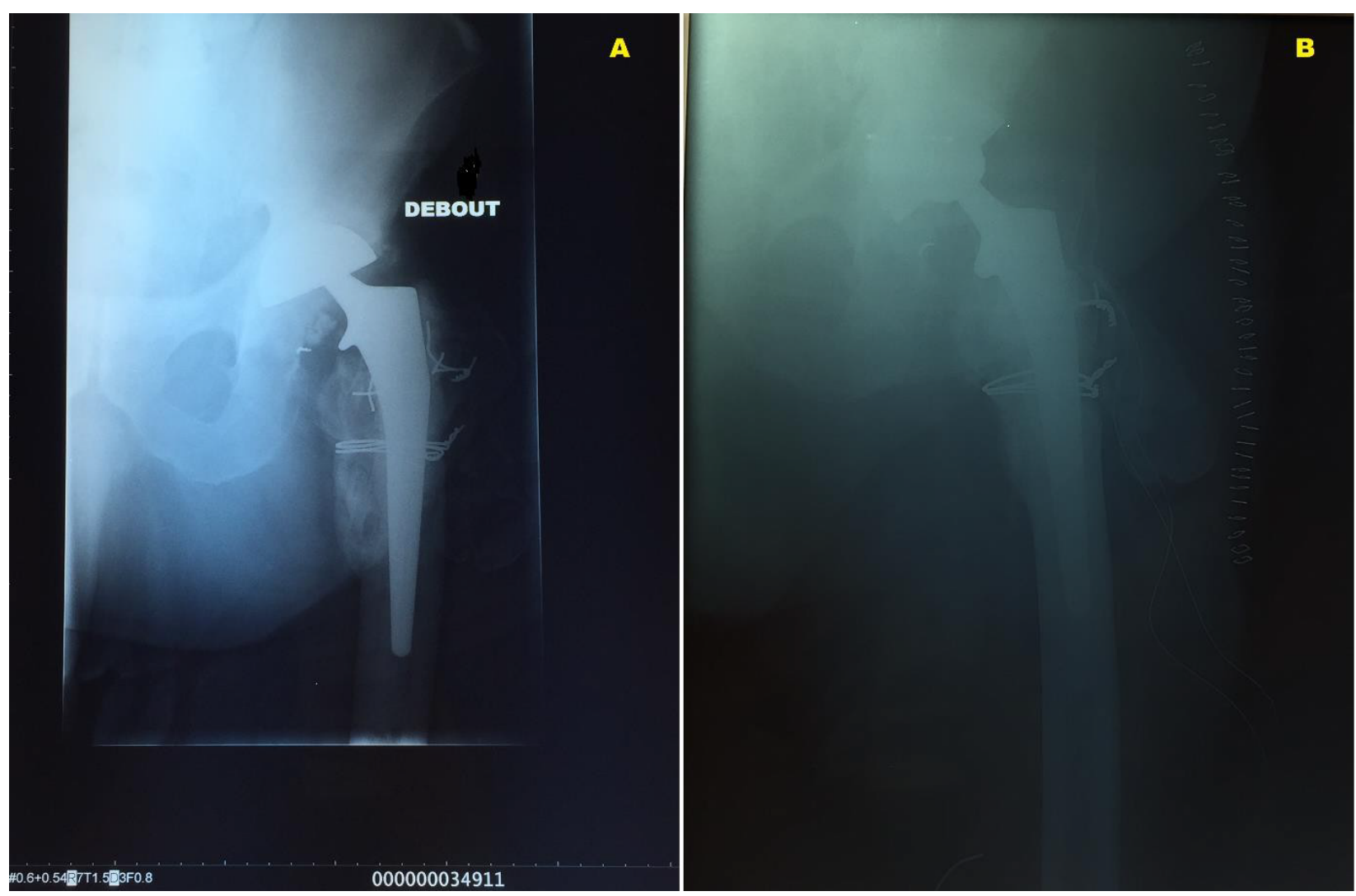

Figure 4: A) The anterior-posterior hip radiograph hip radiograph revealed a protrusion of the prosthetic femoral head through the acetabulum component (fracture of the head femoral or damaged of the polyethylene were suspected); B) postoperative radiograph control showed the normal position of the acetabulum component. 
touching the floor without weight-bearing and transfer onto a wheelchair were permitted 3 weeks postoperatively; partial weight-bearing was permitted at 8 weeks postoperatively, and complete weight bearing with support from a T-cane was allowed 3 months postoperatively. Six months later, the patient regained his usual activities with a good Postel-Merle-d'Aubigné function score.

The patient remained asymptomatic until 5 years when he reported an insidious onset of the progressive left hip pain that is relieved partially with analgesic oral. The evolution was marked by the appearance of clinical lameness requiring the use of crutches that forced the patient to get a visit to our department. At this follow up, with a body mass index of 36 , he reported that his limb has been getting shorter. Physical examination found a shortened left lower extremity of $2 \mathrm{~cm}$; the X-ray of the pelvis showed that the femoral head was embedded in the acetabulum component (Figure 4A). Erythrocyte sedimentation rate and C-reactive protein values were normal. The surgical treatment was established. A few days later, the patient was taken to the operating room; under general anesthetics, an incision was made through the previous scar followed by an appropriate dissection, after which, the Intraoperative aspects showed that the femoral head was slightly damaged and the acetabulum's polyethylene was totally worn; the femoral component was in excellent condition. Implanted components included a dual-mobility acetabular component cemented size 54 and ceramic femoral head with medium neck size $28 \mathrm{~mm}$ (Figure 4B). The patient's initial postoperative course was uncomplicated. At a follow-up of 2 years, the patient was pain-free and could ambulate with no assistive devices and no leg length inequality.

\section{Discussion}

The numbers of revisions of both primary and revision hip implantations are steadily increasing [8]. Revision implantation is more challenging than a primary procedure as the bone stock has often been lost due to stress shielding and the operative removal of the primary implant and the cement mantle, mostly in the proximal region. Many causes of revision surgery are well identified and documented and are regularly studied in the literature [9]. Implant fractures after total hip arthroplasty (THA) are relevant complications for patients, physicians, and the public health system leading to high socioeconomic burdens [10]. When an implant fracture occurred, it is generally an unacceptable complication for the patient and it is not reconcilable with a satisfactory outcome [11]. These fractures occurred between 14 months and 5 years, postoperatively. [12]. Reports in the literature have identified multiple risk factors for revision surgery due to prosthetic stem fracture, including higher levels of activity, relatively younger age, male gender, valgus stem positioning, and relatively undersized stem relative to the patient's anatomy, increased femoral head size, increased femoral offset, and increasing increasing patient weight [13-15]. Currently, Obesity is undeniably a risk factor for poor outcomes and complications after total hip replacement including implant fracture. Almost all cases of implant fractures described in the literature occurred in patients with high BMI $[6,16]$ as in our case.

On the other hand, in revision surgery, the instability of a total hip prosthesis is due to many factors such as age, BMI, ossification, extended liberation of soft tissue, surgical approach and polyethylene wear $[17,18]$. The conventional polyethylene linear and volumetric wear is increased when the acetabular inclination is greater than $45^{\circ}$ [19]. Furthermore, when a diagnosis of isolated polyethylene wear is established, the surgeon must decide whether to exchange the polyethylene alone or revise the acetabular cup instead, especially when he encountered a well-fixed acetabular component. In fact, some authors recommend the complete acetabular revision because the exchange of polyethylene alone increases significantly the risk of instability and dislocation [20] whereas other authors support the exchange of polyethylene alone because of the low dislocation rates in their series and considering that the acetabular revision may cause significant bones losing especially for well-fixed acetabular components [21]. So relying on those factors, there is a question that remains to be answered: Should we have revised the conventional cup, during the first surgery revision, although it was acceptably oriented and well-fixed with no macroscopic wear of the acetabulum's polyethylene? Could this management avoid the second complication? It is still controversial [17].

Although, the dual mobility cup has the great merit to considerably reduce the risk of dislocation rate, and to resist to polyethylene wear in revision surgery, we did not prefer to revise the acetabulum because there was no macroscopic polyethylene wear and the acetabulum was acceptably oriented and well-fixed and so its extraction, re-implantation would be challenging, and it could cause bone loosening that may be difficult to restore.

At last, we emphasize that the experimental study or metallurgical analysis of the broken implant and computed tomography would be helpful to understand well, the exact cause of failure of the primary hip prosthesis. However, we think that the real cause of implant fracture was most probably multifactorial including high BMI, corrosion fatigue of the femoral stem and excessive solicitation of the prosthesis. On the other hand, the revision surgery, high BMI and the inclination of the acetabular less than $35^{\circ}$ were probably the main causes of the acetabulum's polyethylene wear. 
In conclusion, although it is an extremely rare event, implant fracture can be highly traumatizing for patients. The isolated polyethylene wear remains a serious problem for engineers who manufacture prostheses, for surgeons who implant them and for patients who get the result. Finally, we believe that the combination of physical configuration and design of the implant components preoperative risk assessment, optimal surgical technique, and an adequately experienced orthopedic surgeon may promote a good outcome.

\section{Conflict of Interest}

The authors declare no conflict of interest.

\section{Acknowledgements}

Nil.

\section{Sources of Support}

Nil.

\section{Statement of Equal Authors' Contribution}

All authors contribute to the work.

\section{References}

1. Learmonth ID, Young C, Rorabeck C (2007) The operation of the century: Total hip replacement. The Lancet 370: 1508-1519.

2. Chang RW, Pellissier JM, Hazen GB (1996) A Cost-effectiveness analysis of total hip arthroplasty for osteoarthritis of the hip. JAMA 275: 858-865.

3. Kurtz S, Ong K, Lau E, Mowat F, Halpern M (2007) Projections of primary and revision hip and knee arthroplasty in the United States from 2005 to 2030 . J Bone Joint Surg Am 89: 780-785.

4. Kurtz SM, Lau E, Ong K, Zhao K, Kelly M, et al. (2009) Future young patient demand for primary and revision joint replacement: National projections from 2010 to 2030. Clin Orthop Relat Res 467: 2606-2612.

5. Sadoghi $P$, Pawelka W, Liebensteiner MC, Williams A, Leithner A, et al. (2014) The incidence of implant fractures after total hip arthroplasty. Int Orthop 38: 39-46.

6. Al Habsi S, Luthra JS, Al Badei H (2018) Taper fracture of uncemented femoral stem after total hip arthroplasty. Case Rep Orthop Res 1: 50-54.

7. Restrepo C, Ghanem E, Houssock C, Austin M, Parvizi J, et al. (2009) Isolated polyethylene exchange versus acetabular revision for polyethylene wear. Clin Orthop Relat Res 467: 194-198.
8. Kurtz S, Mowat F, Ong K, Chan N, Lau E, et al. (2005) Prevalence of primary and revision total hip and knee arthroplasty in the united states from 1990 through 2002. J Bone Joint Surg Am 87: 1487-1497.

9. Delaunay C, Hamadouche M, Girard J, Duhamel A, SoFCOT Group (2013) What are the causes for failures of primary hip arthroplasties in France? Clin Orthop Relat Res 471: 3863-3869.

10. Furnes A, Lie SA, Havelin LI, Engesaeters LB, Vollset SE (1996) The economic impact of failures in total hip replacement surgery: 28,997 cases from the Norwegian arthroplasty register, 1987-1993. Acta Orthop Scand 67: 115-121.

11. Sadoghi $P$, Liebensteiner $M$, Agreiter $M$, Leithner A, Böhler $\mathrm{N}$, et al. (2013) Revision surgery after total joint arthroplasty: A complication-based analysis using worldwide arthroplasty registers. J Arthroplasty 28: 1329-1332.

12. Carlsson AS, Gentz CF, Stenport J (1977) Fracture of the femoral prosthesis in total hip replacement according to Charnley. Acta Orthop Scand 48: 650-655.

13. Wroblewski BM (1982) Fractured stem in total hip replacement. A clinical review of 120 cases. Acta Orthop Scand 53: $279-284$.

14. Grivas TB, Savvidou OD, Psarakis SA, Bernard PF, Triantafyllopoulos G, et al. (2007) Neck fracture of a cementless forged titanium alloy femoral stem following total hip arthroplasty: A case report and review of the literature. J Med Case Reports 1: 174.

15. McGrory B, Morrey B, Cahalan T, An K, Cabanela M (1995) Effect of femoral offset on range of motion and abductor muscle strength after total hip arthroplasty. J Bone Joint Surg $\mathrm{Br}$ 77: 865-869.

16. ALNohair S (2014) Obesity in Gulf countries. Int J Health Sci (Qassim) 8: 79-83.

17. Leclercq S (2018) Peut-on se passer de la double mobilité dans les révisions de prothèses totales de hanche? 11.

18. Dargel J, Oppermann J, Brüggemann GP, Eysel P (2014) Dislocation following total hip replacement. Dtsch Arztebl Int 111: 884-890.

19. Little NJ, Busch CA, Gallagher JA, Rorabeck CH, Bourne RB (2009) Acetabular polyethylene wear and acetabular inclination and femoral offset. Clin Orthop Relat Res 467: 2895-2900.

20. Blom AW, Astle L, Loveridge J, Learmonth ID (2005) Revision of an acetabular liner has a high risk of dislocation. $J$ Bone Joint Surg Br 87: 1636-1638.

21. O'Brien JJ, Burnett RSJ, McCalden RW, MacDonald SJ, Bourne RB, et al. (2004) Isolated liner exchange in revision total hip arthroplasty: Clinical results using the direct lateral surgical approach. J Arthroplasty 19: 414-423. 